\title{
EVALUACIÓN DE LA SUSTENTABILIDAD DE LA PRODUCCIÓN ORGÁNICA EL CAFÉ A TRAVÉS DE LA MEDICIÓN DE EFICIENCIA ECONÓMICA CON VARIABLES AMBIENTALES
}

Alvarado B., Laura S. ${ }^{1}$

Fecha de recepción: 03-02-13

\section{Resumen}

El estudio evalúa la sustentabilidad de la producción orgánica de café de productores de café en Piura, norte del Perú, a través de la medición de la eficiencia económica que incluya variables ambientales como el balance de nutrientes y el uso del agua agrícola. Se empleó el método de frontera estocástica de producción con el objetivo de comparar la eficiencia económica de la producción orgánica y convencional para cuatro escenarios pertinentes de analizar. Los resultados muestran que no hay mayor diferencia entre la eficiencia económica de ambos tipos de producción. Sin embargo, los ingresos netos o beneficios de los productores orgánicos son mayores a los de los productores convencionales en los cuatro escenarios considerados. Adicionalmente, los costos por balance de nutrientes son menores para la producción orgánica, lo cual indicaría que éste tipo de producción es más sustentable que la convencional.

Palabras Clave: sustentabilidad, eficiencia económica, frontera estocástica de producción, producción orgánica, balance de nutrientes y uso de agua agrícola.

\footnotetext{
Máster en Innovación Agraria para el Desarrollo Rura (UNALM). Consultora en la evaluación económica del Impacto del Cambio Climático en el Perú en el Banco Interamericano de Desarrollo (BID). Profesora auxiliar a dedicación exclusiva (UNALM). Dirección postal: Jr. Valer 595 C Pueblo Libre, LimaPerú. Teléfono: (511) 6147800 anexo: 239; e-mail: lalvarado@ lamolina.edu.pe
}

Fecha de aceptación: 29-08-13

\author{
Clasificación JEL: Q18, Q56.
}

\section{Abstract}

The study aims to assess the sustainability of organic coffee production coffee farmers in Piura, northern Peru, through the measurement of economic efficiency to include environmental variables such as the balance of nutrients and agricultural water use. For this, was used the method of stochastic frontier production with the aim of comparing the economic efficiency of organic and conventional production for four relevant scenarios to analyze. The results show that there is no major difference between the economic efficiency of both types of production. However, net income or profits of organic producers are higher than those of conventional producers in the four scenarios considered. Additionally, costs are lower in nutrient balance for organic production, indicating that this type of production is more sustainable than conventional.

Keywords: sustainability, economic efficien$c y$, stochastic frontier production, organic production, nutrient balance and agricultural water use.

JEL Classification: Q18, Q56. 


\section{INTRODUCCIÓN}

En las últimas décadas, la agricultura orgánica se ha convertido en una de las principales alternativas para la actividad agrícola tradicional y convencional debido al incremento de la demanda de alimentos sanos (libre de insumos químicos) a nivel mundial. Ello ha originado nuevos mercados con precios más atractivos para los agricultores peruanos, muchos de los cuales no tienen acceso a los insumos utilizados para una producción convencional. Así, esta práctica se ha convertido en un mercado significativo cuya demanda por productos a nivel mundial crece a una tasa anual de 10\% (PROMPERU 2008). De este modo, el Perú es un importante exportador de estos productos entre los que destacan, el café, banano, mango, cacao, y castaña entre otros, que generan importantes ingresos. Sin embargo, es necesario no perder de vista la implicancia de esta práctica que es contribuir a la sustentabilidad de la producción agrícola.

Resulta importante, entonces, medir la sustentabilidad con indicadores robustos. La propuesta de esta investigación es medirla a través de la eficiencia económica que incorpore variables ambientales como el balance de nutrientes y el uso del agua. Esto a través de una Frontera Estocástica de Producción y de una Función de Costos que permita obtener la comparación entre la eficiencia económica de la producción convencional y la producción orgánica. Aquella producción que obtenga mayor índice de eficiencia sería más sustentable.

\section{REVISIÓN DE LITERATURA}

\subsection{Sustentabilidad: ¿qué es y cómo medirla?}

Según Bejarano Ávila 1998, citado por Sarandón (2002), el concepto de sustentabilidad ha quedado en la etapa declarativa; y no se ha hecho operativo debido, principalmente, a la dificultad de traducir los aspectos ideológicos de la sustentabilidad en la capacidad de tomar decisiones. Al respecto, Sarandón (2002) señala entre otras razones que esto se debe a la ambigüedad, poca funcionalidad y característica multidimensional del concepto (económica, ecológica, social) y a la ausencia de parámetros comunes de evaluación junto con el uso de herramientas y metodologías adecuadas.

Sobre lo anterior, Simon (2003) también menciona que debido al carácter multidimensional del concepto resulta complicado medirlo. Añade que numerosos indicadores han sido desarrollados simultáneamente, los cuales, o bien destacan los diversos componentes (ecológico, económico, político, social) del concepto por separado (indicadores parciales) o bien encapsulan todos estos componentes a la vez en índices (marcos de indicadores). Cada uno de estos enfoques presenta ventajas y desventajas. Por ejemplo, los indicadores parciales ayudan a entender de una manera más general y compleja el concepto. Sin embargo, la desventaja está en el hecho de que las asociaciones entre las diferentes dimensiones de sustentabilidad (ambiental, 
económica, social e institucional) no están reflejadas a través de dichos indicadores, lo que sí sucede en el caso de los índices.

En este caso, sería más ventajoso emplear un índice. En forma adicional, como esta investigación se centra en el análisis de la sustentabilidad de tecnologías, entendiéndose como tecnologías a la producción convencional y orgánica (o ecológica), conviene discutir sobre qué se entiende por tecnologías sustentables y cómo medir la sustentabilidad de las mismas a través de un índice adecuado.

\subsection{La sustentabilidad de tecnologías: las prácticas orgánicas}

Según Altieri (1994), la agroecología es la base para una producción agrícola sustentable. Plantea que la agricultura moderna enfrenta una crisis ambiental generada por prácticas agrícolas intensivas que se basan en el uso excesivo de insumos que degradan el ambiente y de recursos naturales que conllevan además a la reducción progresiva de la productividad de los cultivos. Ello se evidencia, por ejemplo, en la pérdida de rendimientos por plagas a pesar del aumento de agroquímicos, pues estas se vuelven cada vez más resistentes.

En el Perú, la práctica orgánica en la agricultura se define como una mejora de las condiciones de los suelos y la biodiversidad genética, debido a la utilización racional y óptima de los recursos naturales. Para esta investigación, la producción orgánica es aquella que cuenta con certificación, cuya garantía de serlo son las inspecciones periódicas de parte de las empresas certificadoras.

Para medir la sustentabilidad, Altieri (1994) propone la desagregación de indicadores parciales en parámetros económicos, sociales y biofísicos o ambientales. Entre los económicos, menciona a la dependencia de insumos externos, ingresos, empleo; entre los sociales señala nutrición, viabilidad cultural, aceptabilidad política, equidad; entre los ambientales o biofísicos menciona la productividad del cultivo, rendimientos del suelo, cantidad y calidad del agua para riego, uso de productos químicos en la agricultura. Concluye que la agroecología es aquella que presenta mejores indicadores principalmente ambientales, pues es más sensible a los ciclos naturales y a las interacciones biológicas que la agricultura convencional. Sin embargo, faltan estudios sobre los aspectos socioeconómicos y políticos que más que los problemas técnicos pueden constituirse en barreras para el desarrollo de este tipo de agricultura.

En oposición a Altieri (1994), Sarandón (2002) menciona que diversas tecnologías, inclusive contrapuestas, pueden ser y son promovidas como sustentables. El que sean o no sustentables nadie puede refutarlo o afirmarlo, pues la sustentabilidad no presenta un valor con el cual comparar². A partir de ello, el autor tipifica dos posibilidades de evaluación de la sustentabilidad. La primera la denomina evaluación per se, la cual se centra en contestar

2 Señala por ejemplo que quienes promueven la siembra directa consideran a esta tecnología como sinónimo de sustentabilidad, mientras tanto hay quienes consideran que es todo lo contrario pues promueve un mayor uso de fertilizantes, herbicidas, insecticidas y fungicidas. 
por ejemplo a la pregunta: ¿es sustentable la producción orgánica de café? o ¿es sustentao ble determinada tecnología? Como para estos casos no hay puntos de comparación, entonces exige una respuesta categórica: sí es sustentable o no es sustentable. Sin embargo, para tales respuestas se requiere de un valor absoluto de sustentabilidad lo cual es muy complejo de encontrar.

La segunda posibilidad es la evaluación comparativa de la tecnología, ¿cuál de los dos sistemas es más sustentable? En este caso, no importa el valor absoluto; por lo tanto es más común y fácil de medir. El planteamiento de Sarandón (2002) se centra, entonces, en medir la sustentabilidad a través de un índice que permita comparar cuál de las tecnologías es más sustentable, reafirmando así la propuesta de Simón (2003).

A partir de la revisión, se concluye que la sustentabilidad en la agricultura debe ser medida bajo un criterio comparativo, pues no se puede hablar de la sustentabilidad de una tecnología per se. Esta medición (comparativa) puede realizarse a través de indicadores parciales o índices que capturen la complejidad del concepto (económico, social ambiental). Ambos criterios presentan ventajas y desventajas, sin embargo resulta mejor emplear índices; pues permiten capturar las asociaciones entre las diferentes dimensiones de sustentabilidad (ambiental, económica, social e institucional). El problema, entonces, sería determinar cuál es el mejor índice para medir la sustentabilidad.

\section{3. ¿'Por qué medir la sustentabilidad a través de la eficiencia económica?}

La sustentabilidad implica un manejo de los recursos de tal forma que su abundancia y calidad a largo plazo esté asegurada para las generaciones futuras. En tal sentido, el objetivo de alcanzar un desarrollo sostenible sería un asunto de equidad intergeneracional, sin embargo también es un problema de eficiencia económica, pues reducir la cantidad de recursos naturales (inputs) "por unidad de satisfacción" (outputs) ayudará a reducir la presión sobre el ambiente.

Según lo anterior, se puede decir que la eficiencia económica garantiza parcialmente la existencia de sustentabilidad. Por ello, la investigación la asume como uno de los indicadores más robustos para evaluarla, pues permite incorporar variables ambientales como factores de producción, es decir captura la asociación entre aspectos económicos y ambientales.

Se han encontrado dos trabajos de investigación que miden la sustentabilidad a través de la eficiencia económica. Independientemente del método que emplean para medirla, ya sea Análisis Envolvente de Datos (AED) o Frontera de Estocástica de Producción (FEP), buscan establecer la asociación entre el uso aspectos económicos y ambientales.

Arandía y Aldanondo, en el 2007, investigaron la eficiencia técnica y medioambiental de 
las explotaciones vinícolas ecológicas y las convencionales con el objetivo de analizar y comparar la eficiencia de la producción de uva teniendo en cuenta el impacto ambiental que generan ambas técnicas. Para ello, consideró una muestra de 86 explotaciones agrícolas convencionales y ecológicas. Mediante el $A E D$, se comparó la eficiencia técnica de los dos sistemas de producción sin considerar variables ambientales e incorporando los impactos ambientales. Las explotaciones ecológicas aparecen como más eficientes que las convencionales en todos los casos o escenarios: i) sin considerar costos ambientales, ii) considerando costos ambientales, iii) se toman precios de convencionales y orgánicos y iv) con sólo precios de convencionales. Concluyen que para el primer escenario se debe a que hay un esfuerzo de adaptación que mejora la productividad de los factores y que se trata de agricultura de secano en la que los rendimientos de fertilizantes y pesticidas son menores que en las de regadío. Los resultados para el segundo escenario son razonables $y$, por último, que el efecto del precio sobre la eficiencia es significativo cuando esta se mide sin considerar el impacto ambiental y no lo es cuando se incluyen estos. Es decir, el efecto de la mejora ambiental que introduce la agricultura ecológica parece, por lo tanto, superar al sobreprecio.

Cabrini, Calcaterra y Lema (2011) determinaron el nivel de eficiencia de la producción agrícola en la zona Pergamino (Argentina) para maíz, trigo y soja, considerando los efectos de los servicios ambientales. Si bien no se menciona la medición de la sustentabilidad a través de la eficiencia económica, se incorpo- ra el concepto de servicios ambientales y se incluye su cuantificación dentro de la eficiencia. Los servicios ambientales considerados son los costos y beneficios relacionados al balance de nutrientes del suelo, materia orgánica y la erosión del suelo. Los resultados indican que la actividad agrícola se desarrolla con un alto nivel de eficiencia en la zona de estudio. Se estimó un nivel de eficiencia promedio del $85 \%$. Si bien este valor implica la posibilidad de aumentar un 15\%, la producción para un mismo nivel de insumos, resulta un valor elevado si se lo compara con mediciones realizadas en otros sistemas agrícolas extensivos. No se detectaron relaciones significativas entre el nivel de eficiencia y el nivel de educación, la edad de los responsables de las empresas y la proporción de tierra propia.

\section{MATERIALES $Y$ MÉTODOS}

\section{1. ¿Cómo medir la eficiencia económica?}

Para esta investigación, se empleó el Análisis de Frontera Estocástica (FEP); debido a que no se contaba con la información de series históricas de los diversos factores de producción, sólo se contaría con la información en un momento dado (corte transversal) a partir de la toma de encuesta. El FEP permite hallar la frontera de producción mediante la forma funcional de Cobb-Douglas. Esta forma funcional se eligió, pues permite obtener las elasticidades de los insumos respecto al producto, en este caso particular, respecto a 
la frontera de producción. A partir de la obtención de los estimadores de la función de producción, se puede determinar la eficiencia técnica (ET), la cual se realiza a partir del uso del software Stata 11. Luego, con un modelo de función de costos y sus respectivos estimadores se determina la eficiencia económica (EE), también a partir del uso del software Stata 11 y luego la Eficiencia Asignativa (EA).

El método que se empleó consta de dos etapas. En la primera, se estima la eficiencia técnica, y la económica. En la segunda, la eficiencia asignativa. El modelo estocástico de función de producción para estimar el nivel de eficiencia técnica de las unidades productivas se especifica de la siguiente forma:

$$
Y_{i}=f\left(X_{i} ; \beta\right)+\eta_{i} \quad ; \quad i=1,2,3, \ldots 10 \text { (1) }
$$

Donde $Y_{i}$ es el logaritmo de producción de la i-esima unidad, $X_{i}$ es el vector actual de insumos de la función de producción (en logaritmos) y otras variables relevantes (incluyendo un término constante), mientras que $\beta$ es el vector de parámetros a estimar, y $\eta_{i}$ es el término del error que está compuesto por dos elementos:

$$
\eta_{i}=v_{i}-u_{i}
$$

Donde $V_{i}$ mide las distorsiones simétricas o errores aleatorios que se asumen son independiente e idénticamente distribuidos como $\mathrm{N}\left(0, \delta_{\mathrm{v}}{ }^{2}\right)$, dada la estructura estocástica de una frontera. El segundo componente $u_{i}$ se asume que es independientemente distribuido de $v_{i}$ y se supone que satisface $u_{i} \leq 0$. Para este caso particular, $u_{i}$ se deriva de una distribución $\mathrm{N}\left(0, \delta_{\mathrm{v}}{ }^{2}\right)$, permitiéndole a la producción actual la posibilidad de caer debajo de la frontera.

La ET toma valores dentro del intervalo $(0,1)$, donde 1 indica un predio plenamente eficiente y 0 implica ineficiencia absoluta.

Para cuantificar la eficiencia económica, se especifica una función de costos:

$$
C_{i}=g\left(Y_{i}, P_{i} ; \alpha\right)+\eta_{i} ; i=1,2,3, \ldots 10
$$

Donde $C_{i}$ representa el costo total de producción, $Y_{i}$ la producción producida, $P_{i}$ el costo del insumo, $\alpha$ representa los parámetros de la función de costes, y $\eta_{i}$ representa el término del error, donde:

$$
\eta_{i}=v_{i}-u_{i}
$$

La $E E$ toma valores entre 0 y 1 , donde 1 es cuando el costo efectivo coincide con el costo mínimo, es decir el valor de la función.

Por último, para calcular la Eficiencia de Asignación $(E A)$ se resuelve la siguiente ecuación:

$$
E A=E E / E T
$$

De igual forma, la $E A$ toma el valor de 1 cuando la combinación de factores es la óptima y 0 cuando no lo es. Una vez obtenidos los índices de eficiencia técnica, económica y asignativa por productor convencional y orgánico, se emplean promedios para comparar cuál de las tecnologías presenta mayor índice de eficiencia, es decir está más cercana 
a la combinación óptima de factores.

\section{2. ¿Cómo medir la eficiencia económica incorporando variables ambientales?}

La propuesta de esta investigación es incorporar los factores tradicionales de producción (cantidad de mano de obra, cantidad de fertilizantes,) dos indicadores de sustentabilidad ambiental relevantes: eficiencia en el uso de nutrientes y eficiencia en el uso de agua. $A$ continuación, se detalla el proceso metodológico para obtener ambos indicadores que son incluidos en la función de producción y en la de costos para hallar la eficiencia económica con costos ambientales.

La forma funcional que incorpora variables ambientales en la función de producción se encuentra detallada en el ítem 3.6. Nótese que la función de producción incorpora además de la cantidad de mano de obra y la cantidad de fertilizante, la cantidad de agua (variable ambiental). Mientras que la forma funcional que incorpora variables ambientales en la función de costos se encuentra detallada en el ítem 3.7. Nótese que la función de costos incorpora además del costo por mano de obra y costo por uso de fertilizante, el costo por uso de agua y costo por balance de nutrientes (variables ambientales).

\subsubsection{Eficiencia en el uso de nutrientes}

El estudio de Manchado (2010) respalda esta investigación, pues si bien no emplea una función de producción con frontera esto- cástica, plantea la importancia de emplear y cuantificar, mediante métodos de valoración económica el uso de indicadores para la sustentabilidad en la agricultura como la eficiencia de uso de energía fósil, el riesgo de contaminación por nutrientes o plaguicidas, el riesgo de erosión de suelos, el balance de gases invernadero, la racionalidad en la utilización del suelo, la eficiencia en el uso del agua y el monitoreo de la biodiversidad. Esto debido a que las tendencias en la agricultura moderna como la intensificación del uso del suelo, la ampliación de la frontera agrícola, el uso de fertilizantes han provocado el deterioro de la capacidad de los recursos naturales en su rol de factores productivos, ha puesto en riesgo la sustentabilidad de los sistemas de producción y la ocurrencia de externalidades ambientales.

Manchado (2010) emplea como indicador de sustentabilidad en la agricultura la eficiencia de uso de los nutrientes, es decir el modo en que una especie vegetal, un cultivo o un sistema de producción utilizan los nutrientes. No sólo mide el balance de nutrientes ( $\mathrm{Ni}$ trógeno, Fósforo, Potasio, Magnesio, Azufre y Calcio) en términos físicos sino también los valora económicamente, a través del método de remediación del daño o costos evitados a partir del costo de reposición de los nutrientes mencionados.

Esta investigación asume la propuesta de Manchado (2010), básicamente por la disponibilidad de información. Si bien Cabrini y Calcaterra (2011) proponen la estimación física y la valoración económica del balance de nutrientes, materia orgánica, y erosión hídri- 
ca, no es posible emplear estos dos últimos por la falta de información. Por tal razón, se emplea sólo la valoración de balance de nutrientes según la metodología de Manchado y la propuesta de estimación de eficiencia económica de Cabrini y Calcaterra (2011).

A partir de Manchado (2010), se adaptó la metodología de estimación del balance de nutrientes en términos físicos y monetarios para el caso del café convencional y café orgánico. Los pasos metodológicos fueron los siguientes:

1. Estimación del balance de nutrientes en términos físicos como diferencia entre lo que se exporta (café convencional u orgánico) y lo que se repone por la fertilización.

a. Estimación de la extracción de nutrientes como el producto del coeficiente de extracción de cada nutriente y la producción de café (convencional u orgánico) por individuo. Tal como se muestra:

$$
\text { Ext Nut }_{i j}=\sum c e_{i j}^{*} \operatorname{Prod}_{j}
$$

Donde:

Ext Nut $t_{i j}$ : extracción total de nutrientes para café convencional u orgánico por individuo.

i: extracción de nutrientes (nitrógeno, fósforo, potasio, calcio, magnesio, azufre) $j$ : individuo o productor convencional $u$ orgánico.

$c e_{i j}$ : coeficiente de extracción para cada nutriente de café convencional u orgánico por individuo.

Prod $_{j}$ : producción de café (convencional u orgánico) por individuo.

b. Estimación de la reposición de nutrientes: Para éste caso, Manchado, plantea dos fuentes: a. aplicación de fertilizantes y b. fijación simbiótica. Para esta investigación, se empleó sólo la primera fuente; es decir la aplicación de fertilizantes. Esta información se obtuvo de entrevistas a técnicos, y se consideró la eficiencia de aplicación de fertilizantes de 100\%. La reposición se determina del producto entre macronutriente aportado por fertilizantes por ha. por la cantidad de ha. por productor (convencional u orgánico).

$$
\operatorname{Rep~Nut}_{i j}=\sum \text { fert }_{i j}^{*} \sup _{j}
$$

Donde:

Rep Nut $t_{i j}$ reposición total de nutrientes i: nutriente

$j$ : individuo o productor convencional $u$ orgánico fert $_{i j}$ : nutrientes aportados por fertilización para cada nutriente por productor $\sup _{j}$ : superficie en has por productor convencional u orgánico.

c. Estimación física del balance de nutrientes: Para el cálculo de los balances de nutrientes, se consideraron sólo las extracciones por la exportación de los productos y la reposición de la fertilización. No se consideraron pérdidas por erosión. Se calculó para cada productor 
convencional u orgánico de la siguiente manera:

$$
\text { Bal Nut } t_{i j}=\text { Rep Nut }_{i j}-\text { Ext Nut } t_{i j}
$$$$
\text { (3) }=(2)-(1)
$$

Bal Nut $_{i j}=\sum$ fert $_{i j}^{*} \sup _{j}-\sum c e_{i j}^{*} \operatorname{Prod}_{j}$

2. Valoración económica del balance de nutrientes

Se empleó el método de costo de remediación del daño o costos evitados por la reposición de nutrientes utilizando el fertilizante más usual y de menor precio por unidad de nutriente. Los precios de cada elemento se derivan de los precios corrientes de los fertilizantes en la campaña 2011 (campaña estudiada en la encuesta). Una vez obtenido el costo económico del balance de nutrientes, se incluye en la función de costos para determinar la eficiencia económica.

\subsubsection{Eficiencia en el uso del agua}

Otro indicador importante de sustentabilidad es la eficiencia en el uso de agua. Es importante considerarlo, pues el agua constituye un recurso escaso; $y$, además, no tiene precio en el mercado agrícola en Piura (para el caso del café). Se parte del supuesto que es posible valorar el recurso hídrico (bien que no posee mercado) a través de un bien que sí lo posee (cultivo del café).

Existen diversas metodologías para calcular el valor del agua. Entre éstas, destacan: el método del Cambio de Productividad o una de sus variantes conocidas como el Cambio de los Ingresos Netos del Productor; la Valoración Contingente, el Costo de Oportunidad, el Costo de Conservación y/o Preservación del Acuífero, etc.

El método del Cambio de Productividad, como lo expone Cristeche y Penna (2008), hace posible valorar un bien o servicio ambiental que no se comercializa en el mercado (agua) al relacionarlo con un bien que sí lo hace (cultivos agrícolas). Al considerar que este bien o servicio ambiental es un insumo dentro de la función de producción, la valoración consistiría en evaluar el efecto que dicho bien o servicio ejerce sobre la productividad del cultivo.

Una variante de esta metodología es el Cambio de los Ingresos Netos del Productor. Dicha metodología consiste en considerar al agua como un insumo más en la función de producción de un bien o servicio convencional. El objetivo es estimar el beneficio adicional neto por unidad de agua en la producción de este determinado bien que se transa en el mercado. En este caso, el bien sería el cultivo agrícola (café convencional u orgánico). En tal sentido, el valor económico del agua para uso agrícola se obtiene a través de la siguiente fórmula:

Valor del agua $=\frac{\text { Ingreso neto bajo riego }- \text { Ingreso neto sin irrigación }}{\text { Volumen de agua desviada para irrigación }}$

Donde el ingreso neto es la diferencia entre los ingresos brutos y los costos de producción, cosecha y post-cosecha del café. Se incluye los costos de beneficio húmedo, secado, hasta el transporte y comercialización a la 
planta de CEPICAFE, es decir hasta llegar a un nivel de café pergamino.

Los pasos para realizar la valoración económica del agua para uso agrícola mediante este método se resumen de la siguiente manera:

1. Determinar la cantidad de agua empleada por nivel de productividad de café (convencional u orgánico) que alcanza cada productor. El dato se obtiene de las encuestas.

2. Estimación de la pérdida de productividad de café (convencional u orgánico) por reducción de agua.

Se establece un escenario de reducción probable de agua a través de entrevistas a expertos. Esta reducción de disponibilidad de agua es del 20\%. A partir de esta reducción de agua, se determina la reducción de productividad de café por productor.

3. Valoración económica del agua (VA) determinando ingresos y costos en las situaciones con riego y sin riego a partir de la siguiente fórmula:

$V A=\frac{\left(\left(P * Q_{\text {riego }}\right)-\left(C_{\text {riego }}\right)\right)-\left(\left(P * Q_{\text {sin riego }}\right)-\left(C_{\text {sin riego }}\right)\right)}{V_{\text {agua riego }}}$

Donde:

P: Precio de venta del café convencional u orgánico (S/qq.) pergamino $Q_{\text {riego }}$ : Productividad del café convencional u orgánico con riego (qq/ha)
$Q_{\text {sin riego }}$ Productividad del café convencional u orgánico sin riego ( $q q / \mathrm{ha}$ ) $C_{\text {riego }}$ : Costos de producción del café convencional u orgánico con riego (S/./ha) $C_{\text {sin riego }}:$ Costos de producción del café convencional u orgánico sin riego (S/./ha) $V_{\text {agua riego }}$ :Volumen de agua desviada para la irrigación ( $\mathrm{m}^{3} / \mathrm{ha}$ )

$V A$ : Valor económico del agua para uso agrícola del café convencional u orgánico $\left(\mathrm{S} / . / \mathrm{m}^{3}\right)$

En esta metodología, se comparan dos escenarios: con riego y sin riego (cultivo de secano), siendo el valor del agua igual a la variación de los beneficios entre el agua desviada para riego. En el presente estudio, no se ha limitado por completo el agua de riego durante todo el ciclo del cultivo (cultivo de secano); en cambio, se ha creado el escenario con limitación de agua (reducción del \%20).

Los costos para el escenario con riego y los escenarios proyectados no son iguales. Los costos referentes a los fertilizantes y mano de obra serán incurridos por el agricultor independientemente de la cantidad de agua asignada, pero varían según el nivel de producción. Se asume que el costo de producción se reduce en la misma proporción que el nivel de producción. A pesar que en la zona de estudio, el agua es un recurso escaso, actualmente no existe una tarifa por el uso de agua.

Una vez obtenidos los indicadores de sustentabilidad e incorporados en la función de costos para hallar la eficiencia económica, se contrastan los resultados en los cuatro escenarios relevantes para el análisis. 


\subsection{Población y muestra}

La población está conformada por pequeños productores de tres provincias cafetaleras de la sierra del departamento de Piura pertenecientes a CEPICAFE-Central Piurana de Cafetaleros, con un tamaño menor a 3 ha. La población total es de 1943 productores de los cuales 1203 son orgánicos y 730 son convencionales. El principal criterio para diferenciar a productores orgánicos de convencionales es el hecho de contar con certificación orgánica. Para hallar el tamaño de muestra se empleó la fórmula de poblaciones finitas siendo el tamaño de 160 observaciones, desagregadas entre 60 productores convencionales y 100 productores orgánicos. El Nivel de Error es de 0.075 , mientras que el nivel de confianza es del $92,5 \%$.

\subsection{Sobre los factores de producción de la población analizada}

El estudio ha considerado como factores de producción el uso de fertilizantes, la mano de obra y el uso de agua. Las maquinarias son artesanales y dan cuenta de un bajo nivel tecnológico para ambos casos (café convencional y orgánico), por ello no fueron relevantes al momento del análisis.

En cuanto a los abonos y fertilizantes, los productores orgánicos (es decir los que cuentan con certificación orgánica) utilizan el guano de isla, sulfomag, roca fosfórica y fertimar. Cada uno de estos cuatro insumos contiene los seis macronutrientes analizados para el balance de nutrientes: Nitrógeno, Fósforo, Potasio, Calcio, Magnesio y Azufre. Los productores convencionales se caracterizan por usar fertilizantes químicos o no permitidos en la producción orgánica, por no emplear ningún tipo de fertilizante o por emplear los fertilizantes usados por los orgánicos pero en dosis inferiores o inapropiadas. Esto va ligado al menor acceso a servicios de asistencia técnica que tienen. La mano de obra es más intensa para el caso de productores orgánicos pues se requiere mayores labores.

El uso de agua es otro factor importante. El nivel tecnológico en el riego es limitado. EI 93\% de los productores emplean el riego por gravedad, sólo el 7\% emplean el riego por aspersión. El agua es un factor importante en la producción y también en la post-cosecha, pues se requiere para el lavado del café. El estudio ha considerado el uso total de agua para todo el proceso de producción de café pergamino. Son los productores orgánicos los que demandan más agua en el proceso de producción, seguramente por la mayor exigencia en el beneficio del café (el 77\% emplea más de 3000 m3, frente a un $28 \%$ de los convencionales), según se observa en el Anexo 1.

\subsection{Diseño metodológico}

Primero se realizan las estimaciones de Frontera Estocástica a través de las funciones de producción para café convencional y orgánico, luego se determina también a través de Fronteras Estocásticas las Funciones de costo para estimar la eficiencia económica bajo cuatro escenarios: i) sin costos ambientales y 
ii) con costos ambientales a precios de producto convencional y orgánico y iii) sin costos ambientales y iv) con costos ambientales a precios de producto convencional y finalmente se presentan las conclusiones.

\subsection{Estimaciones de función de producción a través de Fronteras Estocásticas}

Se empleó el siguiente modelo para ambas funciones: de café convencional y de café orgánico. La estimación de esta función permite hallar la eficiencia técnica de ambos tipos de producción. Las variables especificadas como una función de tipo Cobb-Douglas, son las siguientes:

$$
l y_{i}=\beta_{1} l x 1_{i}+\beta_{2} l x 2_{i}+\beta_{3} l x 3_{i}+\left(v_{i}-u_{i}\right)
$$

Donde:

$l y_{i}=$ Logaritmo del rendimiento del café convencional en $\mathrm{kg} / \mathrm{ha}$ del productor $\mathrm{i}$

lx $1_{i}=$ Logaritmo de la cantidad de fertilizantes en $\mathrm{kg} / \mathrm{ha}$ del productor $\mathrm{i}$

$\mathrm{l} x 2_{i}=$ Cantidad de mano de obra en jornales/ ha del productor $\mathrm{i}$

$\mathrm{lx} 3_{i}=$ Cantidad de agua en $\mathrm{m}^{3} /$ ha del productor $\mathrm{i}$

$\left(v_{i}-u_{i}\right)=$ término del error compuesto por dos elementos

$v_{i}=$ Componente aleatorio, que captura los errores de medición y otros factores

$u_{i}=$ Componente aleatorio no negativo que representa el nivel de ineficiencia del productor i que sigue una distribución normal no negativa.

\subsection{Estimaciones de la función de costos usando Fronteras Estocásticas}

A continuación, se plantean las estimaciones de la función de costos usando el método de Fronteras Estocásticas. El objetivo de estas es encontrar la eficiencia económica para ambos tipos de café (convencional y orgánico) en los cuatro escenarios establecidos: i) sin costos ambientales y con precios de café convencional y orgánico; ii) con costos ambientales a precios de café orgánico y convencional; iii) sin costos ambientales a precios de café convencional; iv) con costos ambientales a precios de café convencional. Como ya se ha mencionado, los costos ambientales implican los costos por uso de agua y el costo de reparación por balance de nutrientes. El análisis de precios de café convencional consistió en emplear un precio promedio de café convencional para evitar la distorsión por el diferencial existente para el caso del café orgánico. A continuación, se presentan los modelos empleados para cada escenario:

\section{Escenario 1: Sin costos ambientales a pre- cios de café orgánico y convencional}

$$
l y_{i}=\beta_{1} l x 1_{i}+\beta_{2} l x 2_{i}+\beta_{3} l x 3_{i}+\beta_{4} l x 4_{i}^{*}+e_{i}
$$

Donde:

$l y_{i}=$ Ingreso neto en soles/ha del productor $\mathrm{i}$ $l x 1_{i}=$ Superficie en ha del productor $\mathrm{i}$ $\mathrm{l} x 2_{i}=$ Costo del jornal en soles del productor $\mathrm{i}$ $1 \times 3_{i}=$ Costo del fertilizante en soles del productor $\mathrm{i}$ 
$\mathrm{l} x 4_{i}=$ Rendimiento ajustado en $\mathrm{kg} / \mathrm{ha}$ para el productor $\mathrm{i}$

$e_{1}=$ Error idiosincrático.

\section{Escenario 2: Con costos ambientales a pre-} cios de café orgánico y convencional

$$
\begin{gathered}
l y_{i}=\beta_{0}+\beta_{1} l x 1_{i}+\beta_{2} l x 2_{i}+\beta_{3} l x 3_{i}+\beta_{3} l x 4_{i}+ \\
\beta_{3} l \times 5_{i}+\beta_{4} l x 6_{i}^{*}+e_{i}
\end{gathered}
$$

Donde:

$l y_{i}=$ Ingreso neto en soles/ha del productor $\mathrm{i}$ $I \times 1_{i}=$ Superficie en ha del productor $\mathrm{i}$

$1 \times 2_{i}=$ Costo del jornal en soles del productor $\mathrm{i}$ $\operatorname{lx} 3_{i}=$ Costo del fertilizante en soles del productor $\mathrm{i}$

$l_{X} 4_{i}=$ Costo de balance de nutrientes para el productor $\mathrm{i}$

$l x 5_{i}=$ Costo de agua en soles por $\mathrm{m}^{3} /$ ha para el productor $\mathrm{i}$

${ } \mathrm{x} \sigma_{i}=$ Rendimiento ajustado en $\mathrm{kg} / \mathrm{ha}$ para el productor $\mathrm{i}$

$e_{i}=$ Error idiosincrático.

Escenario 3: Sin costos ambientales a precios de café convencional

$l_{i}=\beta_{0}+\beta_{1} l \times 1_{i}+\beta_{2} l \times 2_{i}+\beta_{3} l x 3_{i}+\beta_{4} l \times 4_{i}^{*}+e_{i}$

Donde:

$l y_{i}=$ Ingreso neto en soles/ha del productor $\mathrm{i}$ $I_{x} 1_{i}=$ Superficie en ha del productor $\mathrm{i}$ $\mathrm{Ix} 2_{i}=$ Costo del jornal en soles del productor $\mathrm{i}$ $I \times 3_{i}=$ Costo del fertilizante en soles del productor $\mathrm{i}$ $\mathrm{l} \times 4_{i}=$ Rendimiento ajustado en $\mathrm{kg} / \mathrm{ha}$ para el productor $\mathrm{i}$

$e_{i}=$ Error idiosincrático

Escenario 4: Con costos ambientales a precios de café convencional

$$
\begin{gathered}
l y_{i}=\beta_{0}+\beta_{1} l x 1_{i}+\beta_{2} l x 2_{i}+\beta_{3} l \times 3_{i}+\beta_{3} l x 4_{i}+ \\
\beta_{3} l \times 5_{i}+\beta_{4} l x 6_{i}^{*}+e_{i}
\end{gathered}
$$

Donde:

$l y_{i}=$ Ingreso neto en soles/ha del productor i $\operatorname{lx} 1_{i}=$ Superficie en ha del productor $\mathrm{i}$ $\operatorname{lx} 2_{i}=$ Costo del jornal en soles del productor $\mathrm{i}$ $l \times 3_{i}=$ Costo del fertilizante en soles del productor i

$\mathrm{IX}_{i}=$ Costo de balance de nutrientes para el productor $\mathrm{i}$

$l \times 5_{i}=$ Costo de agua en soles por $\mathrm{m}^{3} /$ ha para el productor $\mathrm{i}$

$l x \sigma_{i}=$ Rendimiento ajustado en $\mathrm{kg} / \mathrm{ha}$ para el productor $\mathrm{i}$

$e_{i}=$ Error idiosincrático.

Para cada escenario, nótese que $1 \times 4_{i}{ }^{*}$ y $l \times 6_{i}{ }^{*}$ representan el rendimiento ajustado. Éste es resultado de la diferencia del Rendimiento en $\mathrm{kg} / \mathrm{ha}$ menos la ineficiencia técnica $u_{i}$ (sigma_u) obtenido en las estimaciones anteriores. En este estudio, se generaron dos tipos de rendimiento ajustado. El primero es de la diferencia del rendimiento menos la ineficiencia técnica que se generó sin la inclusión de la cantidad de fertilizantes y el otro tipo es la diferencia del rendimiento menos la ineficiencia técnica que se generó con la inclusión de la cantidad de fertilizantes. Así, el error idiosincrático está asociado a la cantidad de fertilizantes. Por lo tanto, este rendimiento 
ajustado ocasionó que para cada escenario existan dos estimaciones. Este procedimiento se desarrolló para productores convencionales y orgánicos.

\section{RESULTADOS}

\subsection{Eficiencia técnica}

En las corridas econométricas para determinar la función de producción, se emplearon como variables dependientes la producción (medido en $\mathrm{kg}$ ) y el rendimiento ( $\mathrm{kg} / \mathrm{ha}$ ). Como resultado, se obtuvo como mejor variable dependiente al rendimiento. Para el café convencional, el mejor modelo (es decir aquel que presenta mayor nivel de significancia o $R^{2}$ ) fue aquel que incluye las variables fertilizantes, mano de obra y agua. En cambio para el café orgánico, resultó mejor el modelo que incluye sólo mano de obra y agua, y no incluye fertilizante. Lo anterior se justifica, porque las cantidades de insumos orgánicos empleadas en la producción son mínimas.

Asimismo, las eficiencias técnicas fueron 64\% y $63 \%$ para el café convencional y orgánico, respectivamente. No se encontró variación significativa entre una tecnología y otra, según se observa en el Anexo 2.

\subsection{Valoración económica de variables ambientales}

Los resultados de la valoración económica del balance de nutrientes y del uso del agua se muestran en el Anexo 3. Se pudo apreciar que los productores orgánicos presentan un menor costo que los productores convencionales por balance de nutrientes ( $\mathrm{S} / 99$ /ha y $S / 110 /$ ha respectivamente). En cambio, no se apreció por el valor del agua ( $S / 714 /$ ha y S/254/ha, respectivamente).

Los costos por balance de nutrientes y por uso agrícola del agua fueron incorporados en los costos totales y en los ingresos netos o valor neto de la producción. Así, se analizan los resultados de los costos en dos escenarios: sin considerar costos ambientales y considerando costos ambientales. Según se observa en el Anexo 4, los costos para ambos escenarios son mayores para el caso de productores orgánicos, esto debido al uso de fertilizantes permitidos que incrementan los costos.

Los ingresos netos o beneficios se muestran para cuatro escenarios: i) sin costos ambientales y con precios de café convencional y orgánico; ii) con costos ambientales a precios de café orgánico y convencional; iii) sin costos ambientales a precios de café convencional; iv) con costos ambientales a precios de café convencional. El análisis de precios de café convencional consistió en emplear un precio promedio de café convencional a fin de evitar la distorsión por el diferencial existente para el caso del café orgánico.

Según se observa, también en el Anexo 4, los ingresos netos para los productores orgánicos son mayores a los de los productores convencionales en los cuatro escenarios. De esta manera, se puede observar que sin considerar variables ambientales a precios de café orgánico y convencional (escenario 1) los ingre- 
sos netos de los productores orgánicos son significativamente mayores que los ingresos de los productores convencionales. En el escenario 2, los ingresos también son mayores para los productores orgánicos, a pesar que se reducen significativamente con respecto al escenario 1 (se reducen a la mitad), sin embargo la diferencia se incrementa, a pesar que los costos para los productores orgánicos con mayores en todos los casos. Realizando el análisis a precios de café convencional (para evitar distorsiones con respecto a sobreprecios), los ingresos netos de los productores orgánicos no se ven afectados, porque en las últimas campañas los precios diferenciales se han acortado considerablemente, tanto así que para muchos productores de café orgánico le da igual vender su producto a precio de café convencional.

Los productores orgánicos presentan un menor costo que los productores convencionales por balance de nutrientes ( $S / 99 /$ ha y $S / 110 /$ ha respectivamente); no así por el valor del agua (S/714/ha y $S / 254 /$ ha respectivamente). Esto se puede deber a que los valores obtenidos para el uso del agua agrícola hacen referencia sólo a costos por cantidad de agua, sin embargo el valor no representa los costos por reducción de la calidad de agua o a la erosión hídrica del suelo.

Si se empleara una metodología que incorpore la pérdida por calidad de agua, los costos se podrían incrementar, probablemente en mayor medida en los productores convencionales que en los productores orgánicos; ya que las prácticas orgánicas exigen emplear técnicas para uso eficiente del agua como por ejemplo los pozos de miel.

Las variables ambientales incluidas en el análisis si bien incrementan aun más los costos de la producción orgánica (pues son mayores que los costos de producción convencional), no afectan negativamente los ingresos netos o beneficios de los productores orgánicos que en todos los escenarios presentan mayores valores que los productores convencionales. De esta manera, se puede afirmar que los ingresos netos de los productores orgánicos son mayores que los ingresos netos de los productores convencionales, incluso eliminando el diferencial por precio.

\subsection{Eficiencia económica}

Con respecto a la eficiencia económica, no hay diferencias sustanciales para los casos de productores orgánicos y convencionales. Sin embargo, la eficiencia económica para productores convencionales es ligeramente mayor en un escenario sin considerar variables ambientales (escenario 1). En el escenario 2, la eficiencia económica para productores orgánicos es mayor, mientras que para el escenario 3, la eficiencia económica es igual para ambos tipos de productores y en escenario ,4 la eficiencia económica de productores orgánicos es mayor a la de los productores convencionales, según se observa en el Anexo 5.

\section{CONCLUSIONES}

A partir de los resultados, se concluye: 


\section{Sobre las relaciones entre sustenta- bilidad, innovación tecnológica y efi- ciencia económica}

El análisis de la sustentabilidad y la innovación tecnológica de agricultura convencional a agricultura orgánica se centra en tres puntos: la productividad; los cambios en la calidad ambiental y los efectos en los precios. A partir de estos cuatro temas, se puede concluir que: i) la productividad de la agricultura orgánica de la zona estudiada no es menor que la de la agricultura convencional; ii) la evidencia muestra que los indicadores ambientales en términos físicos no son necesariamente mejores para la agricultura convencional y iii) la producción orgánica permite obtener un sobreprecio a los agricultores lo cual evidencia una mayor disposición para pagar por parte de los consumidores.

El principal argumento para medir la sustentabilidad del cambio de producción convencional a orgánica, a través de la eficiencia económica, es que si bien la agricultura orgánica podría contribuir hacia un manejo más racional de los recursos naturales, ello no debe ser medido solamente desde el punto de vista ambiental basado en el argumento que tendría un menor impacto de la actividad sobre el medio ambiente, sino que debe ser medido económicamente; pues es necesario tener presente que se trata de una actividad productiva y como tal debería ser rentable para el productor.

Para eliminar el supuesto de una posible mayor eficiencia de la producción orgánica por el sobreprecio, se midió la eficiencia económica en cuatro escenarios: sin costos ambientales, con costos ambientales, con precios convencionales y orgánicos y sólo con precios convencionales.

La eficiencia económica se midió por el método de FEP, e incluye variables ambientales como balance de nutrientes y uso de agua las cuales se valoran económicamente. Los valores económicos de dichas variables ambientales fueron incluidos en la función de costos para determinar la eficiencia económica. Esta metodología se considera relevante por ser una adaptación de varios estudios revisados, y por no haberse realizado previamente.

\section{Sobre los ingresos netos de los pro- ductores orgánicos son mayores que los ingresos netos de los productores convencionales, incluso eliminando el diferencial por precio}

Se apreció que los productores orgánicos presentaron un menor costo que los productores convencionales por balance de nutrientes no en cambio por el valor del agua. Los costos por balance de nutrientes y por uso agrícola del agua fueron incorporados en los costos totales y en los ingresos netos o valor neto de la producción. Se analizaron los resultados de los costos en dos escenarios: sin considerar costos ambientales y considerando costos ambientales. Los costos para ambos escenarios son mayores para el caso de productores orgánicos, esto debido al uso de fertilizantes permitidos que incrementan los costos. Los ingresos netos o beneficios se muestran para cuatro escenarios: i) sin costos ambientales y con precios de café conven- 
cional y orgánico; ii) con costos ambientales a precios de café orgánico y convencional; iii) sin costos ambientales a precios de café convencional; iv) con costos ambientales a precios de café convencional. Las variables ambientales incluidas en el análisis, si bien incrementan aun más los costos de la proo ducción orgánica (pues son mayores que los costos de producción convencional), no afectan negativamente los ingresos netos o beneficios de los productores orgánicos que en todos los escenarios presenta mayores valores que los productores convencionales. De esta manera, se comprueba la hipótesis que los ingresos netos de los productores orgánicos son mayores que los ingresos netos de los productores convencionales, incluso eliminando el diferencial por precio.

\section{Sobre la producción orgánica es más eficiente económicamente que la producción convencional de café}

Con respecto a la eficiencia económica, no hay diferencias sustanciales para los casos de productores orgánicos y convencionales, así no se puede afirmar que la producción orgánica sea más eficiente que la convencional. En otras palabras, no se puede afirmar que la producción orgánica emplee una combinación más óptima en el uso de factores que la producción convencional.

La eficiencia económica para productores convencionales es, sin embargo, ligeramente mayor en un escenario sin considerar variables ambientales (escenario 1). En el escenario 2, la eficiencia económica para productores orgánicos es mayor, mientras que para el escenario 3, la eficiencia económica es igual para ambos tipos de productores y en escenario 4 la eficiencia económica de productores orgánicos es mayor a la de los productores convencionales. Los costos ambientales (por balance de nutrientes y por uso agrícola del agua) influyen en el incremento de los costos para los productores orgánicos en mayor proporción que para los productores convencionales. Lo anterior explicaría una eficiencia económica no muy diferenciada entre ambos tipos de productores. Sin embargo, haciendo un análisis de ingresos netos o beneficios se constata que éstos para los productores orgánicos son mayores a los de los productores convencionales en los cuatro escenarios.

Cabe señalar que el costo por el uso del agua es el que incrementa los costos de la producción orgánica, por tanto si se toma en cuenta sólo la variable balance de nutrientes la eficiencia económica del café orgánica sería mayor a la convencional. La recomendación es que se invierta en tecnología de riego para emplear óptimamente el agua en la producción orgánica.

Por último, es necesario destacar que la valoración económica de los ingresos han sido medidos sólo por venta de café en el mercado y no, por ejemplo, por externalidades positivas de la producción orgánica como conservación de biodiversidad o captura de carbono, lo cual hubiera implicado el uso de otras metodologías más complejas. Por ello, se recomiendan estudios posteriores para incorporar este análisis. 


\section{REFERENCIAS BIBLIOGRÁFICAS}

Altieri M. (1994). Bases Agroecológicas para una producción agrícola sustentable. Agricultura Técnica Chile, 74 (4): 371-386. Octubre-Diciembre 1994.

Arandía A, Aldanondo A. (2007). Eficiencia Técnica y medioambiental de las explotaciones vinícolas ecológicas versus convencionales. Revista Española de Estudios Agrosociales y Pesqueros Número 215-216, (115-184). 2007.

Bell S, Morse S. (2008). Sustainability indicators. Measuring the immeasurable? Second Edit tion, 2008.

Cabrini S, Calcaterra C y Lema D. (2011). Costos ambientales y eficiencia productiva en la producción agraria del partido de Pergamino. INTA, 2011.

Coelli TJ, Prasada R, O'Donnell CJ, Battese GE. (1998). An introduction to efficiency and productivity analysis, second edition. 1998

Cristeche E., Penna J. (2008) Métodos de valoración económica de los servicios ambientales. ISSN 1851-6955. Instituto Nacional de Tecnología Agropecuaria. Argentina.2008.

Izac MN, Swift MJ. (1994). On agricultural sustainability and its measurement in small-scale farming in sub-Saharan Africa. 1994
Manchado, J. (2010). La sustentabilidad en la agricultura pampeana: Valoración económica del balance de nutrientes para las principales actividades agropecuarias extensivas en la Región Centro Sur de la Provincia de Buenos Aires.2010.

PROMPERU. (2008). Producción orgánica en el Perú y sus perspectivas. Agosto 2008. Consultado en http://www.siicex.gob.pe/siicex/ resources/sectoresproductivos/d546ec3cb220-4396-a2a7-a509812a8e31.pdf el 18 de Marzo de 2011.

Sarandón SJ. (2002). Agroecologia: El camino hacia una agricultura sustentable", SJ Sarandón (Editor), Ediciones Científicas Americanas, La Plata. 560 pp. 2002

Simon S. (2003). Sustainability Indicators. In: Neumayer, Eric ed. Online Encyclopaedia of Ecological Economics (OEEE). International Society for Ecological Economics (ISEE). 


\section{ANEXOS}

Anexo 1. Productores de café según tipo de riego y uso de agua

\begin{tabular}{|l|c|c|c|c|c|c|}
\hline \multirow{2}{*}{ Productor } & \multicolumn{3}{|c|}{ Tipo de riego } & \multicolumn{3}{c|}{ Uso de agua en m3 } \\
\cline { 2 - 7 } & Gravedad & Aspersión & Total & $\begin{array}{c}\text { Menor igual } \\
\text { a 3000 m3 }\end{array}$ & $\begin{array}{c}\text { Mayor a } \\
\mathbf{3 0 0 0} \text { m3 }\end{array}$ & Total \\
\hline Convencional & $92 \%$ & $8 \%$ & $100 \%$ & $72 \%$ & $28 \%$ & $100 \%$ \\
Orgánico & $94 \%$ & $6 \%$ & $100 \%$ & $23 \%$ & $77 \%$ & $100 \%$ \\
Total & $93 \%$ & $7 \%$ & $100 \%$ & $41 \%$ & $59 \%$ & $100 \%$ \\
\hline
\end{tabular}

Fuente: Elaboración propia sobre la base de procesamiento de datos encuesta.

Anexo 2. Eficiencia técnica para producción convencional y orgánica

\begin{tabular}{|l|c|c|c|c|}
\hline \multicolumn{1}{|c|}{ Producción } & Media & Desviación típica & Mínimo & Máximo \\
\hline Convencional & 0.64 & 0.21 & 0.32 & 1 \\
Orgánica & 0.63 & 0.19 & 0.18 & 0.93 \\
\hline
\end{tabular}

Fuente: Elaboración propia.

Anexo 3. Costos ambientales por balance de nutrientes y uso de agua agrícola para el café según tipo de productor en $\mathrm{S} / \mathrm{ha}$

\begin{tabular}{|c|c|c|c|c|c|c|c|c|}
\hline \multirow[b]{2}{*}{ Costos (S/ha) } & \multicolumn{4}{|c|}{ Productores convencionales } & \multicolumn{4}{|c|}{ Productores orgánicos } \\
\hline & Media & Máximo & Mínimo & $\begin{array}{l}\text { Desviación } \\
\text { típica }\end{array}$ & Media & Máximo & Mínimo & $\begin{array}{c}\text { Desviación } \\
\text { típica }\end{array}$ \\
\hline $\begin{array}{l}\text { Costo del balance de } \\
\text { nutrientes }\end{array}$ & 110 & 135 & 45 & 21 & 99 & 194 & 12 & 54 \\
\hline Costo del uso de agua & 254 & 2227 & 0 & 442 & 714 & 3447 & 0 & 658 \\
\hline
\end{tabular}

Fuente: Elaboración propia. 


\section{Anexo 4. Costos e ingresos netos con y sin considerar variables ambientales (S/ha)}

\begin{tabular}{|c|c|c|c|c|c|c|c|c|c|}
\hline \multirow[b]{2}{*}{ Escenario } & \multirow{2}{*}{$\begin{array}{l}\text { Costos e in- } \\
\text { gresos (s/ha) }\end{array}$} & \multicolumn{4}{|c|}{ Productores convencionales } & \multicolumn{4}{|c|}{ Productores orgánicos } \\
\hline & & Media & Máximo & Mínimo & $\begin{array}{l}\text { Desviación } \\
\text { típica }\end{array}$ & Media & Máximo & Mínimo & $\begin{array}{c}\text { Desviación } \\
\text { típica }\end{array}$ \\
\hline Escenario 1 & $\begin{array}{c}\text { Costos totales sin } \\
\text { variables ambientales }\end{array}$ & 767 & 2104 & 284 & 346 & 1670 & 4691 & 454 & 755 \\
\hline Escenario 2 & $\begin{array}{c}\text { Costos totales con } \\
\text { variables ambientales }\end{array}$ & 1131 & 3066 & 431 & 578 & 2483 & 8203 & 582 & 1144 \\
\hline Escenario 1 & $\begin{array}{l}\text { Ingresos netos sin } \\
\text { variables ambientales }\end{array}$ & 483 & 3807 & -1224 & 983 & 1659 & 5577 & -1371 & 1770 \\
\hline Escenario 2 & $\begin{array}{l}\text { Ingresos netos con } \\
\text { variables ambientales }\end{array}$ & 119 & 3168 & -1343 & 721 & 846 & 4378 & -1467 & 1325 \\
\hline Escenario 3 & $\begin{array}{l}\text { Ingresos netos sin } \\
\text { variables a precios } \\
\text { de café convencional }\end{array}$ & 471 & 3637 & -1252 & 912 & 1731 & 5596 & -1357 & 1667 \\
\hline Escenario 4 & $\begin{array}{l}\text { Ingresos netos con } \\
\text { variables ambientales } \\
\text { a precios de café } \\
\text { convencional }\end{array}$ & 107 & 3512 & -1371 & 643 & 918 & 4182 & -1426 & 1230 \\
\hline
\end{tabular}

Fuente: Elaboración propia.

\section{Anexo 5. Eficiencia económica en los cuatros escenarios para productores de café convencional y orgánico}

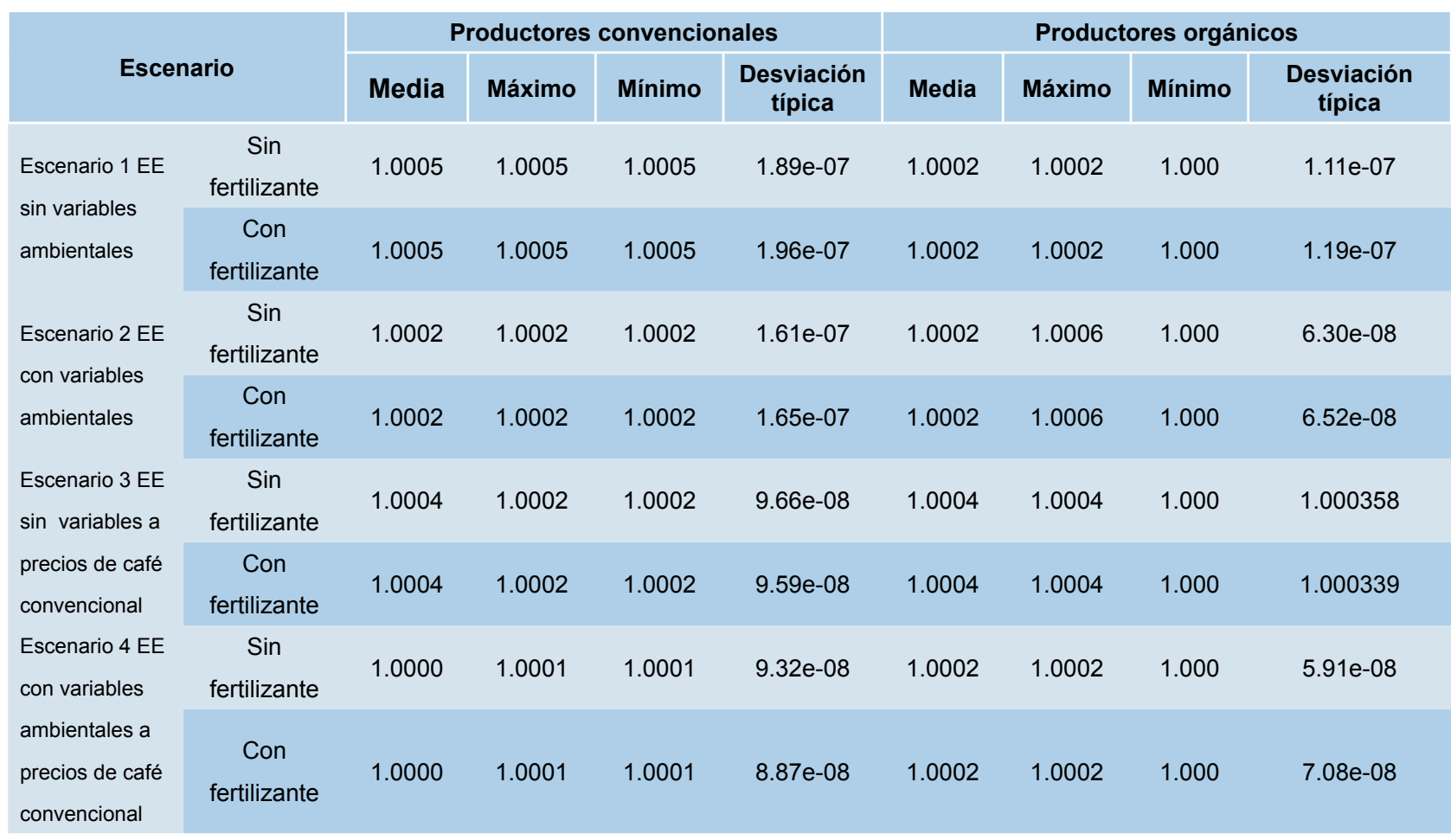

Fuente: Elaboración propia. 